QUARTERLY OF APPLIED MATHEMATICS

VOLUME LXVII, NUMBER 1

MARCH 2009, PAGES 73-92

S 0033-569X(08)01117-4

Article electronically published on December 22, 2008

\title{
THE HELMHOLTZ EQUATION IN THE EXTERIOR OF SLITS IN A PLANE WITH DIFFERENT IMPEDANCE BOUNDARY CONDITIONS ON OPPOSITE SIDES OF THE SLITS
}

\author{
BY \\ P. A. KRUTITSKII \\ KIAM, Department 25, Miusskaya Square 4, Moscow 125047, Russia
}

\begin{abstract}
The boundary value problem for the Helmholtz equation is studied outside slits in a plane. The impedance boundary conditions are specified on the slits. In general, the impedance conditions may be different at different sides of each slit. In a particular case, the impedance conditions may be the same on the sides of each slit. It is proved that the classical solution to the problem exists, and it is unique. The integral representation for a solution to the problem is obtained in the form of potentials, the densities in which are uniquely determined from the uniquely solvable system of the Fredholm integral equations of the second kind and index zero.
\end{abstract}

1. Introduction. The Dirichlet and Neumann problems for the Laplace and Helmholtz equations in the exterior of several single-sided open arcs in a plane have been studied in $[9,21,22$. If we consider each open arc as a slit in a plane, then by singlesided open arcs we mean the case when the same boundary data is specified on both sides of the slits.

In general, problems in the exterior of several slits in a plane imply that different boundary data is specified on different sides of the slits. Boundary value problems for the Helmholtz equation in the exterior of several slits in a plane describe scattering of acoustic waves by several cylindrical double-sided screens in fluids or by several cracks in solids. Dirichlet and Neumann problems in the exterior of several slits in a plane have been studied for the Helmholtz equation in [1]-2], the mixed problem with setting Dirichlet and Neumann boundary conditions on different slits has been studied in [3], and the boundary conditions, containing jumps of the solutions or its normal derivatives have been studied in $[4-[5$. The boundary value problems in $1,-5]$ were reduced to the uniquely solvable integral equations, and for each problem the integral representation for a solution has been obtained in the form of potentials.

Received July 1, 2007.

2000 Mathematics Subject Classification. Primary 35J05, 35J25, 45E05, 76 Q05.

(C)2008 Brown University 
The present paper is devoted to the analysis of the boundary value problem for the Helmholtz equation in the exterior of several slits in a plane, when the impedance boundary conditions are specified on different sides of each slit. The impedance boundary conditions may be different on different sides of each slit. The particular case of this problem with simplified boundary conditions has been studied in [20]. The problem studied in the present paper describes propagation and scattering of acoustic waves in a fluid with soft acoustic screens. The problem is very important for engineering applications, for example, the numerical solution of its particular case for one slit has been obtained in 7]. However the general case has not been treated by methods of analysis before. Theorems of existence and uniqueness of a solution are obtained in the present paper for the general case of the impedance problem in the exterior of several slits in a plane. The integral representation for a solution is obtained in the form of potentials. The densities in potentials are found by solving the Fredholm equation of the second kind and index zero. It is shown that the Fredholm equation is uniquely solvable in a certain Banach space. Singularities of the gradient of a solution are studied at the ends of the slits. The theory of numerical methods for singular integral equations appearing in this problem is developed in [8, p. 366].

2. Formulation of the problem. By a simple open curve we mean a non-closed smooth arc of finite length without self-intersections [9, p. 13]. Consider simple open curves $\Gamma_{1}, \ldots, \Gamma_{N}$, which belong to $C^{2, \lambda}, \lambda \in(0,1]$, and have no common points (including end points), in a plane $x=\left(x_{1}, x_{2}\right) \in R^{2}$. Denote $\Gamma=\bigcup_{n=1}^{N} \Gamma_{n}$. Assume that each curve $\Gamma_{n}$ is parametrized by the arc length $s$ :

$$
\Gamma_{n}=\left\{x: \quad x=x(s)=\left(x_{1}(s), x_{2}(s)\right), \quad s \in\left[a_{n}, b_{n}\right]\right\}, \quad n=1, \ldots, N,
$$

so that $a_{1}<b_{1}<\ldots<a_{N}<b_{N}$. Then points $x \in \Gamma$ and values of the parameter $s$ are in one-to-one correspondence. Let $\Gamma$ denote the set of the segments $\bigcup_{n=1}^{N}\left[a_{n}, b_{n}\right]$ of the $O s$ axis as well as the contour $\Gamma$. Let $\tau_{x}=(\cos \alpha(s), \sin \alpha(s))$ be a tangent vector to $\Gamma$ at the point $x(s)$ in the direction of growth of the parameter $s$. Let $\mathbf{n}_{x}=(\sin \alpha(s),-\cos \alpha(s))$ denote the normal vector to $\Gamma$ at the point $x(s)$ which coincides with the tangent vector $\tau_{x}$ after rotation by the angle $\pi / 2$ counter-clockwise. Owing to chosen parametrization $\cos \alpha(s)=x_{1}^{\prime}(s), \sin \alpha(s)=x_{2}^{\prime}(s)$. Let the plane $R^{2}$ be slit along the contour $\Gamma$. Denote the side of the slits $\Gamma$ which remains on the left while parameter $s$ increases by $\Gamma^{+}$, and the opposite side by $\Gamma^{-}$.

We say that the function $u(x)$ belongs to the smoothness class $\mathbf{K}$ if the following conditions are satisfied:

1) $u(x) \in C^{0}\left(\overline{R^{2} \backslash \Gamma}\right) \cap C^{2}\left(R^{2} \backslash \Gamma\right)$, in particular, $u(x)$ is continuous at the ends of $\Gamma$;

2) $\nabla u \in C^{0}\left(\overline{R^{2} \backslash \Gamma} \backslash X\right)$, where $X$ is a set of end points of $\Gamma$ :

$$
X=\bigcup_{n=1}^{N}\left(x\left(a_{n}\right) \cup x\left(b_{n}\right)\right) ;
$$


3) if $x \rightarrow x(d) \in X$ and $x \notin \Gamma$, then the inequality

$$
|\nabla u(x)|<C|x-x(d)|^{\varepsilon}
$$

holds, where the constant $C>0$, the number $\varepsilon>-1$ and $d=a_{n}$ or $d=b_{n}$ for $n=1, \ldots, N$.

REMARK. The class of functions continuous in $R^{2} \backslash \Gamma$ and continuously extendible from the left and right to $\Gamma \backslash X$ is denoted by $C^{0}\left(\overline{R^{2} \backslash \Gamma} \backslash X\right)$. The limiting values of these functions on $\Gamma \backslash X$ from the left can be different, so these functions may have a jump if one passes across $\Gamma \backslash X$. Functions of class $C^{0}\left(\overline{R^{2} \backslash \Gamma}\right)$ possess the same properties, but, in addition, they are continuously extendible to the ends of the slits $\Gamma$.

Let us formulate a boundary value problem for the Helmholtz equation in $R^{2} \backslash \Gamma$.

Problem $\mathbf{U}$. Find a function $u(x)$ of the class $\mathbf{K}$ satisfying the Helmholtz equation in $R^{2} \backslash \Gamma$

$$
\triangle u+k^{2} u=0, \quad k=\text { const } \neq 0, \quad 0 \leq \arg k<\pi,
$$

and the impedance boundary conditions

$$
\begin{aligned}
& \left.\frac{\partial u}{\partial \mathbf{n}}\right|_{x(s) \in \Gamma^{+}}+\left.\beta_{1}(s) u(x)\right|_{x(s) \in \Gamma^{+}}=f_{1}(s), \\
& \left.\frac{\partial u}{\partial \mathbf{n}}\right|_{x(s) \in \Gamma^{-}}-\left.\beta_{2}(s) u(x)\right|_{x(s) \in \Gamma^{-}}=f_{2}(s),
\end{aligned}
$$

where the functions $f_{1}(s), f_{2}(s), \beta_{1}(s), \beta_{2}(s)$ are specified on $\Gamma$, and the $\beta_{j}(s)$ satisfy one of the following additional conditions for $j=1,2$ :

1. If $k=\operatorname{Re} k>0$, then $\operatorname{Im} \beta_{j}(s) \leq 0$ for any $s \in \Gamma$.

2. If $\operatorname{Re} k=0$ and $\operatorname{Im} k>0$, then $\beta_{j}(s)=\operatorname{Re} \beta_{j}(s) \geq 0$ for any $s \in \Gamma$.

3. If $\operatorname{Re} k \neq 0, \operatorname{Im} k>0$, then $(\operatorname{Re} k) \cdot\left(\operatorname{Im} \beta_{j}(s)\right) \leq 0$ for any $s \in \Gamma$.

In addition, the function $u(x)$ must satisfy the conditions at infinity. If $\arg k=0$, i.e. $k=\operatorname{Re} k>0$, then we set the Sommerfeld conditions at infinity:

$$
u(x)=O\left(\frac{1}{\sqrt{|x|}}\right), \quad \frac{\partial u(x)}{\partial|x|}-i k u(x)=o\left(\frac{1}{\sqrt{|x|}}\right), \quad|x| \rightarrow \infty .
$$

If $0<\arg k<\pi$, i.e. $\operatorname{Im} k>0$, then we set the following conditions at infinity:

$$
u(x)=o\left(\frac{1}{\sqrt{|x|}}\right), \quad \frac{\partial u(x)}{\partial|x|}=o\left(\frac{1}{\sqrt{|x|}}\right), \quad|x|=\sqrt{x_{1}^{2}+x_{2}^{2}} \rightarrow \infty .
$$

All conditions of the problem are regarded in the classical sense. The validity of the boundary conditions (3) at the ends of $\Gamma$ is not required. The condition (1) at the ends of $\Gamma$ in the definition of the class $\mathbf{K}$ ensures the absence of the point sources at the ends of $\Gamma$. If $\beta_{1}(s) \equiv \beta_{2}(s)$, then Problem $\mathbf{U}$ transforms to the problem studied in [20]. The Neumann problem in the exterior of slits in a plane [2] is a particular case of [20].

Let $k=\operatorname{Re} k>0$ (or $\operatorname{Re} k \neq 0, \operatorname{Im} k>0$ ), and let $\operatorname{Im} \beta_{1}(s) \equiv \operatorname{Im} \beta_{2}(s) \equiv 0$, i.e. $\beta_{1}(s)$, $\beta_{2}(s)$ are real functions. If $\beta_{1}(s) \equiv-\beta_{2}(s)$, then the boundary condition (3b) takes the 
form

$$
\left.\frac{\partial u}{\partial \mathbf{n}}\right|_{x(s) \in \Gamma^{-}}+\left.\beta_{1}(s) u(x)\right|_{x(s) \in \Gamma^{-}}=f_{2}(s),
$$

so the left sides in the boundary conditions (3a) and (3b) coincide. Therefore, the same boundary condition is posed on opposite sides of the slits in this particular case of Problem $\mathbf{U}$, but the boundary data $f_{1}(s)$ and $f_{2}(s)$ on opposite sides of the slits can be different.

REMARK. The boundary conditions (3) can be written in the form:

$$
\begin{array}{r}
\left.\frac{\partial u}{\partial \mathbf{n}}\right|_{x(s) \in \Gamma^{+}}+\left.\frac{\partial u}{\partial \mathbf{n}}\right|_{x(s) \in \Gamma^{-}} \\
+\left(\left.\beta_{1}(s) u(x)\right|_{x(s) \in \Gamma^{+}}-\left.\beta_{2}(s) u(x)\right|_{x(s) \in \Gamma^{-}}\right)=f_{1}(s)+f_{2}(s), \\
\left.\frac{\partial u}{\partial \mathbf{n}}\right|_{x(s) \in \Gamma^{+}}-\left.\frac{\partial u}{\partial \mathbf{n}}\right|_{x(s) \in \Gamma^{-}} \\
+\left(\left.\beta_{1}(s) u(x)\right|_{x(s) \in \Gamma^{+}}+\left.\beta_{2}(s) u(x)\right|_{x(s) \in \Gamma^{-}}\right)=f_{1}(s)-f_{2}(s) .
\end{array}
$$

The boundary conditions $\left(3 \mathrm{a}^{\prime}\right)$ and $\left(3 \mathrm{~b}^{\prime}\right)$ are equivalent to the boundary conditions (3a) and $(3 \mathrm{~b})$.

Theorem 1. If $\Gamma \in C^{2, \lambda}, \lambda \in(0,1], \beta_{1}(s), \beta_{2}(s) \in C^{0}(\Gamma)$, then there is no more than one solution to Problem U.

Proof. Let $u_{0}(x)$ be a solution to the homogeneous Problem $\mathbf{U}$. We will prove that $u_{0}(x) \equiv 0$ by the method of integral equalities. Let $C_{r}$ be a disc containing $\Gamma$ of radius $r$ with the center in the origin. Consider a set of $N$ simple smooth closed curves $\Lambda$ enveloping the contour $\Gamma$. We assume that curves $\Lambda$ have no common points and each curve envelops only one slit $\Gamma_{n}$. Writing out first Green's formula [10, p. 328] for the function $u_{0}(x)$ in a domain bounded by $\Lambda$ and $\partial C_{r}$, taking into account that $u_{0}(x) \in \mathbf{K}$, shrinking $\Lambda$ to $\Gamma$ and then setting $r \rightarrow \infty$, we obtain

$$
\begin{gathered}
\lim _{r \rightarrow \infty}\left(\left\|\nabla u_{0}\right\|_{L_{2}\left(C_{r} \backslash \Gamma\right)}^{2}-k^{2}\left\|u_{0}\right\|_{L_{2}\left(C_{r} \backslash \Gamma\right)}^{2}\right)=\int_{\Gamma} \bar{u}_{0}^{+}\left(\frac{\partial u_{0}}{\partial \mathbf{n}_{x}}\right)^{+} d s \\
-\int_{\Gamma} \bar{u}_{0}^{-}\left(\frac{\partial u_{0}}{\partial \mathbf{n}_{x}}\right)^{-} d s+\left\{\begin{array}{c}
i k \lim _{r \rightarrow \infty} \int_{\partial C_{r}}\left|u_{0}\right|^{2} d l, \quad \text { in case of conditions (4a), } \\
0, \quad \text { in case of conditions (4b), }
\end{array}\right.
\end{gathered}
$$

where conditions (1), (4) were used. The complex conjugate function to $u_{0}(x)$ is denoted by $\bar{u}_{0}(x)$. Clearly, $\bar{u}_{0}(x) \in \mathbf{K}$. The superscripts + and - denote the limiting values of the functions on $\Gamma^{+}$and $\Gamma^{-}$respectively. Here and further on we use the designation $\int_{\Gamma} \ldots d s=\sum_{n=1}^{N} \int_{a_{n}}^{b_{n}} \ldots d s$.

REMARK. It follows from section 1.5 of the book [6, pp. 24-25] that any solution to equation (2) satisfying radiating conditions (4a) has the following asymptotic behavior 
at infinity:

$$
u_{0}(x)=\frac{e^{i k r}}{\sqrt{r}} \mathcal{F}_{0}(\phi)+O\left(\frac{1}{r^{3 / 2}}\right), \quad r=|x| \rightarrow \infty,
$$

where $\phi$ is a polar angle, and $\mathcal{F}_{0}(\phi)$ is a continuous function. This asymptotic formula proves the existence of the limit

$$
\lim _{r \rightarrow \infty} \int_{\partial C_{r}}\left|u_{0}\right|^{2} d l=\int_{0}^{2 \pi}\left|\mathcal{F}_{0}(\phi)\right|^{2} d \phi
$$

in the right side (5a) in case of conditions (4a). Therefore, the limit in the left side of (5a) exists as well.

Using homogeneous boundary conditions (3) for the function $u_{0}(x)$, we write the equality (5a) in the form

$$
\begin{gathered}
\lim _{r \rightarrow \infty}\left(\left\|\nabla u_{0}\right\|_{L_{2}\left(C_{r} \backslash \Gamma\right)}^{2}-k^{2}\left\|u_{0}\right\|_{L_{2}\left(C_{r} \backslash \Gamma\right)}^{2}\right) \\
=-\int_{\Gamma} \beta_{1}(s)\left|u_{0}^{+}(x(s))\right|^{2} d s-\int_{\Gamma} \beta_{2}(s)\left|u_{0}^{-}(x(s))\right|^{2} d s \\
+\left\{\begin{array}{cc}
i k \lim _{r \rightarrow \infty} \int_{\partial C_{r}}\left|u_{0}\right|^{2} d l, \quad \text { in case of conditions (4a) }, \\
0, \quad \text { in case of conditions (4b). }
\end{array}\right.
\end{gathered}
$$

If $\operatorname{Re} k=0$ and $\operatorname{Im} k>0$, so that $k^{2}=-|k|^{2}$, then conditions (4b) are satisfied and we obtain

$$
\begin{gathered}
\lim _{r \rightarrow \infty}\left(\left\|\nabla u_{0}\right\|_{L_{2}\left(C_{r} \backslash \Gamma\right)}^{2}+|k|^{2}\left\|u_{0}\right\|_{L_{2}\left(C_{r} \backslash \Gamma\right)}^{2}\right) \\
=-\int_{\Gamma}\left(\left|\beta_{1}(s)\right| \cdot\left|u_{0}^{+}(x(s))\right|^{2}+\left|\beta_{2}(s)\right| \cdot\left|u_{0}^{-}(x(s))\right|^{2}\right) d s,
\end{gathered}
$$

since in this case $\operatorname{Re} \beta_{j}(s)=\beta_{j}(s) \geq 0$ for any $s \in \Gamma$ and $j=1,2$. Hence $\left\|u_{0}\right\|_{L_{2}\left(R^{2} \backslash \Gamma\right)}^{2}=0$, so $u_{0}(x) \equiv 0$. Further on we assume that $\operatorname{Re} k \neq 0$ and $\operatorname{Im} k>0$ in case of conditions (4b). As noted above, limits in (5b) exist. Consequently they exist for both real and imaginary parts. Choosing the imaginary part in (5b), we obtain

$$
\begin{aligned}
& -\int_{\Gamma}\left(\left(\operatorname{Im} \beta_{1}(s)\right)\left|u_{0}^{+}(x(s))\right|^{2}+\left(\operatorname{Im} \beta_{2}(s)\right)\left|u_{0}^{-}(x(s))\right|^{2}\right) d s \\
& +k \lim _{r \rightarrow \infty} \int_{\partial C_{r}}\left|u_{0}\right|^{2} d l=0 \quad \text { for conditions (4a), } \\
& -\int_{\Gamma}\left(\left(\operatorname{Im} \beta_{1}(s)\right)\left|u_{0}^{+}(x(s))\right|^{2}+\left(\operatorname{Im} \beta_{2}(s)\right)\left|u_{0}^{-}(x(s))\right|^{2}\right) d s \\
& +\operatorname{Im} k^{2} \lim _{r \rightarrow \infty}\left\|u_{0}\right\|_{L_{2}\left(C_{r} \backslash \Gamma\right)}^{2}=0 \quad \text { for conditions (4b), }
\end{aligned}
$$

where we used that $k=\operatorname{Re} k>0$ in case of conditions (4a) and, in addition, $\operatorname{Re} k \neq 0$, $\operatorname{Im} k>0$ in case of conditions (4b). In accordance with the formulation of Problem $\mathbf{U}$, 
if $\operatorname{Re} k \neq 0$ and $\operatorname{Im} k>0$, then $\left(\operatorname{Im} \beta_{j}(s)\right) \cdot(\operatorname{Re} k) \leq 0$ for any $s \in \Gamma$ and $j=1,2$, whence $-\left(\operatorname{Im} \beta_{j}(s)\right) /\left(\operatorname{Im} k^{2}\right) \geq 0$, since $\operatorname{Im} k^{2}=2 \operatorname{Re} k \cdot \operatorname{Im} k \neq 0$. In addition, it is required in Problem $\mathbf{U}$ that $\operatorname{Im} \beta_{1}(s) \leq 0$ and $\operatorname{Im} \beta_{2}(s) \leq 0$ for any $s \in \Gamma$ if $k=\operatorname{Re} k>0$. Therefore, equalities $(5 \mathrm{c})$ can be written in the form

$$
\begin{aligned}
& \frac{1}{|k|} \int_{\Gamma}\left(\left|\operatorname{Im} \beta_{1}(s)\right| \cdot\left|u_{0}^{+}(x(s))\right|^{2}+\left|\operatorname{Im} \beta_{2}(s)\right| \cdot\left|u_{0}^{-}(x(s))\right|^{2}\right) d s \\
& \quad+\lim _{r \rightarrow \infty} \int_{\partial C_{r}}\left|u_{0}\right|^{2} d l=0 \quad \text { in case of conditions (4a), } \\
& \frac{1}{\left|\operatorname{Im} k^{2}\right|} \int_{\Gamma}\left(\left|\operatorname{Im} \beta_{1}(s)\right| \cdot\left|u_{0}^{+}(x(s))\right|^{2}+\left|\operatorname{Im} \beta_{2}(s)\right| \cdot\left|u_{0}^{-}(x(s))\right|^{2}\right) d s \\
& +\lim _{r \rightarrow \infty}\left\|u_{0}\right\|_{L_{2}\left(C_{r} \backslash \Gamma\right)}^{2}=0 \quad \text { in case of conditions (4b). }
\end{aligned}
$$

Hence

$$
\begin{gathered}
\lim _{r \rightarrow \infty} \int_{\partial C_{r}}\left|u_{0}\right|^{2} d l=0 \quad \text { in case of conditions (4a), } \\
\lim _{r \rightarrow \infty}\left\|u_{0}\right\|_{L_{2}\left(C_{r} \backslash \Gamma\right)}^{2}=0 \quad \text { in case of conditions (4b). }
\end{gathered}
$$

If $k=\operatorname{Re} k>0$ and conditions (4a) hold, then $u_{0}(x) \equiv 0$ owing to the Rellich lemma [11, p. 682]. If $\operatorname{Re} k \neq 0, \operatorname{Im} k>0$ and conditions (4b) hold, then $\left\|u_{0}\right\|_{L_{2}\left(R^{2} \backslash \Gamma\right)}^{2}=0$, i.e. $u_{0}(x) \equiv 0$. Thus, in all cases $u_{0}(x)$ is a trivial solution to the homogeneous Problem $\mathbf{U}$. Therefore there exists only the trivial solution to the homogeneous Problem $\mathbf{U}$, and the theorem is proved in view of the linearity of Problem $\mathbf{U}$.

3. Reduction of the problem to the integral equations. Denote the Hankel function of the first kind and zero order [12, p. 332] by $\mathcal{H}_{0}^{(1)}(z)$ :

$$
\mathcal{H}_{0}^{(1)}(z)=\frac{\sqrt{2} \exp (i z-i \pi / 4)}{\pi \sqrt{z}} \int_{0}^{\infty} \exp (-t) t^{-1 / 2}\left(1+\frac{i t}{2 z}\right)^{-1 / 2} d t .
$$

To construct the solution to Problem $\mathbf{U}$, we assume that the functions $\beta_{1}(s), \beta_{2}(s), f_{1}(s)$, $f_{2}(s)$ in the boundary conditions (3) belong to the Hölder space:

$$
\beta_{1}(s), \beta_{2}(s), f_{1}(s), f_{2}(s) \in C^{0, \lambda}(\Gamma), \quad \lambda \in(0,1] .
$$

Consider the angular potential [1, 2] on $\Gamma$ for Equation (2):

$$
v\left[\mu_{1}\right](x)=\frac{i}{4} \int_{\Gamma} \mu_{1}(\sigma) V(x, \sigma) d \sigma .
$$

The kernel $V(x, \sigma)$ is given on each curve $\Gamma_{n} \quad(n=1, \ldots, N)$ by the formula

$$
V(x, \sigma)=\int_{a_{n}}^{\sigma} \frac{\partial \mathcal{H}_{0}^{(1)}(k|x-y(\xi)|)}{\partial \mathbf{n}_{y}} d \xi, \quad \sigma \in\left[a_{n}, b_{n}\right],
$$

where $y=y(\xi)=\left(y_{1}(\xi), y_{2}(\xi)\right), \quad|x-y(\xi)|=\sqrt{\left(x_{1}-y_{1}(\xi)\right)^{2}+\left(x_{2}-y_{2}(\xi)\right)^{2}}$. 
Further on we will assume that $\mu_{1}(\sigma)$ belongs to the Banach space $C_{q}^{\omega}(\Gamma)$ for some $\omega$, $q$, such that $\omega \in(0,1], q \in[0,1)$.

We say that $\mu_{1}(s)$ belongs to the Banach space $C_{q}^{\omega}(\Gamma)$ with $\omega \in(0,1], q \in[0,1)$, if $\mu_{1}(s) \prod_{n=1}^{N}\left|s-a_{n}\right|^{q}\left|s-b_{n}\right|^{q} \in C^{0, \omega}(\Gamma)$, where $C^{0, \omega}(\Gamma)$ is a space of Hölder continuous functions with the exponent $\omega$. The norm in the Banach space $C_{q}^{\omega}(\Gamma)$ is determined by the relation $\left\|\mu_{1}(s)\right\|_{C_{q}^{\omega}(\Gamma)}=\left\|\mu_{1}(s) \prod_{n=1}^{N}\left|s-a_{n}\right|^{q}\left|s-b_{n}\right|^{q}\right\|_{C^{0, \omega}(\Gamma)}$.

In addition, further on we will assume that $\mu_{1}(s)$ satisfies the following conditions [1, 2]:

$$
\int_{a_{n}}^{b_{n}} \mu_{1}(\sigma) d \sigma=0, \quad n=1, \ldots, N
$$

As was shown in [1,2], the angular potential $v\left[\mu_{1}\right](x)$ with such a density $\mu_{1}(\sigma)$ belongs to the class $\mathbf{K}$. In particular, inequality (1) holds for $\varepsilon=-q$ if $q \in(0,1)$ and for any $\varepsilon \in(-1,0)$ if $q=0$. Besides, integrating $v\left[\mu_{1}\right](x)$ by parts and using $(8)$, we represent the angular potential in the form of the double layer potential

$$
v\left[\mu_{1}\right](x)=-\frac{i}{4} \int_{\Gamma} \rho\left[\mu_{1}\right](\sigma) \frac{\partial \mathcal{H}_{0}^{(1)}(k|x-y(\sigma)|)}{\partial \mathbf{n}_{y}} d \sigma
$$

with the density

$$
\rho\left[\mu_{1}\right](\sigma)=\int_{a_{n}}^{\sigma} \mu_{1}(\xi) d \xi, \quad \sigma \in\left[a_{n}, b_{n}\right], \quad n=1, \ldots, N .
$$

Consequently, $v\left[\mu_{1}\right](x)$ satisfies both equation (2) in the exterior of $\Gamma$ and the conditions at infinity (4).

We shall construct a solution to Problem $\mathbf{U}$ with the help of potential theory for the Helmholtz equation (2). We shall look for a solution to the problem in the form of a sum of an angular potential and a single layer potential

$$
u\left[\mu_{1}, \mu_{2}\right](x)=v\left[\mu_{1}\right](x)+w\left[\mu_{2}\right](x),
$$

where $v\left[\mu_{1}\right](x)$ is an angular potential defined in $(7),(9)$, and

$$
w\left[\mu_{2}\right](x)=\frac{i}{4} \int_{\Gamma} \mu_{2}(\sigma) \mathcal{H}_{0}^{(1)}(k|x-y(\sigma)|) d \sigma
$$

is a single layer potential. We shall look for the function $\mu_{2}(s)$ in the space $C^{0, \lambda / 4}(\Gamma)$. It was mentioned above that we look for $\mu_{1}(s)$ in the Banach space $C_{q}^{\omega}(\Gamma), \omega \in(0,1], q \in$ $[0,1)$. In addition, the density $\mu_{1}(s)$ must satisfy conditions (8). For such densities $\mu_{1}(s)$ and $\mu_{2}(s)$ the function (11) belongs to the class $\mathbf{K}$ (see [1]) and satisfies all conditions of Problem U, except for the boundary conditions (3). In particular, the function (11) satisfies conditions at infinity (4).

Consider boundary conditions in the form of $\left(3 \mathrm{a}^{\prime}\right)$ and $\left(3 \mathrm{~b}^{\prime}\right)$. To satisfy boundary conditions $\left(3 \mathrm{a}^{\prime}\right),\left(3 \mathrm{~b}^{\prime}\right)$, we substitute (11) into $\left(3 \mathrm{a}^{\prime}\right)$, $\left(3 \mathrm{~b}^{\prime}\right)$, use formulas for limiting 
values of the angular potential from [1] and arrive at the integral equations for densities $\mu_{1}(s)$ and $\mu_{2}(s)$ :

$$
\begin{gathered}
-\frac{1}{\pi} \int_{\Gamma} \mu_{1}(\sigma) \frac{\sin \varphi_{0}(x(s), y(\sigma))}{|x(s)-y(\sigma)|} d \sigma+\frac{i}{2} \int_{\Gamma} \mu_{1}(\sigma) \frac{\partial V_{0}(x(s), \sigma)}{\partial \mathbf{n}_{x}} d \sigma \\
+\frac{i}{2} \int_{\Gamma} \mu_{2}(\sigma) \frac{\partial \mathcal{H}_{0}^{(1)}(k|x(s)-y(\sigma)|)}{\partial \mathbf{n}_{x}} d \sigma+\frac{1}{2}\left(\beta_{1}(s)+\beta_{2}(s)\right) \rho\left[\mu_{1}\right](s) \\
+\left(\beta_{1}(s)-\beta_{2}(s)\right) \frac{i}{4}\left(\int_{\Gamma} \mu_{2}(\sigma) \mathcal{H}_{0}^{(1)}(k|x(s)-y(\sigma)|) d \sigma+\int_{\Gamma} \mu_{1}(\sigma) V(x(s), \sigma) d \sigma\right) \\
\mu_{2}(s)+\left(\beta_{1}(s)+f_{1}(s)+f_{2}(s), \quad s \in \Gamma, \quad{ }^{i}\left(\int_{\Gamma} \mu_{2}(\sigma) \mathcal{H}_{0}^{(1)}(k|x(s)-y(\sigma)|) d \sigma+\int_{\Gamma} \mu_{1}(\sigma) V(x(s), \sigma) d \sigma\right)\right. \\
+\frac{1}{2}\left(\beta_{1}(s)-\beta_{2}(s)\right) \rho\left[\mu_{1}\right](s)=f_{1}(s)-f_{2}(s), \quad s \in \Gamma,
\end{gathered}
$$

where $\rho\left[\mu_{1}\right](s)$ is defined in (10),

$$
\begin{gathered}
V_{0}(x, \sigma)=\int_{a_{n}}^{\sigma} \frac{\partial h(k|x-y(\xi)|)}{\partial \mathbf{n}_{y}} d \xi, \quad \sigma \in\left[a_{n}, b_{n}\right], \quad n=1,2, \ldots, N, \\
h(z)=\mathcal{H}_{0}^{(1)}(z)-\frac{2 i}{\pi} \ln \frac{z}{k} .
\end{gathered}
$$

The angle between the vector $\overrightarrow{x y}$ and the direction of the normal $\mathbf{n}_{x}$ is denoted by $\varphi_{0}(x, y)$. The angle $\varphi_{0}(x, y)$ is positive if it is measured from the vector $\mathbf{n}_{x}$ counter-clockwise, and negative if it is measured from the vector $\mathbf{n}_{x}$ clockwise. Moreover, the angle $\varphi_{0}(x, y)$ is continuous if $x, y \in \Gamma$ and $x \neq y$.

The first term in (12) is a singular Cauchy integral [9, p. 315]. Let us study properties of the operator $\rho\left[\mu_{1}\right](s)$ from (10). Since the function $\mu_{1}(s)$ belongs to $C_{q}^{\omega}(\Gamma)$, it can be represented in the form $\mu_{1}(s)=\mu_{1 *}(s) \mathcal{Q}_{q}^{-1}(s)$, where $\mu_{1 *}(s) \in C^{0, \omega}(\Gamma)$, and

$$
\mathcal{Q}_{q}(s)=\prod_{n=1}^{N}\left|s-a_{n}\right|^{q}\left|b_{n}-s\right|^{q} \operatorname{sign}\left(s-a_{n}\right), \quad q \in[0,1), \quad s \in \Gamma .
$$

Consider the operator

$$
\rho_{q}\left[\mu_{1 *}\right](s)=\rho\left[\mu_{1}\right](s)=\int_{a_{n}}^{s} \mu_{1 *}(\sigma) \mathcal{Q}_{q}^{-1}(\sigma) d \sigma, \quad s \in\left[a_{n}, b_{n}\right], \quad n=1, \ldots, N .
$$

Lemma 1. (a) The operator $\rho_{q}\left[\mu_{1 *}\right](s)$ with $q \in[0,1)$ is bounded when it acts from $C^{0}(\Gamma)$ into $C^{0,1-q}(\Gamma)$.

(b) The operator $\rho\left[\mu_{1}\right](s)$ is bounded when it acts from $C_{q}^{\omega}(\Gamma)$ with $\omega \in(0,1]$ and $q \in[0,1)$ into $C^{0,1-q}(\Gamma)$. 
Proof. The proof is demonstrated in [5].

Denote

$$
A_{22}^{ \pm}(s, \sigma)=\frac{i}{4}\left(\beta_{2}(s) \pm \beta_{1}(s)\right) \mathcal{H}_{0}^{(1)}(k|x(s)-y(\sigma)|) .
$$

Rewrite the Cauchy singular integral equation (12) in the form

$$
\begin{gathered}
\frac{1}{\pi} \int_{\Gamma} \mu_{1}(\sigma) \frac{d \sigma}{\sigma-s}+\int_{\Gamma} \mu_{1}(\sigma) Y_{11}(s, \sigma) d \sigma+\int_{\Gamma} \mu_{2}(\sigma) Y_{12}(s, \sigma) d \sigma+Y\left[\mu_{2}\right](s) \\
-\frac{1}{2}\left(\beta_{1}(s)+\beta_{2}(s)\right) \rho\left[\mu_{1}\right](s)=-\left(f_{1}(s)+f_{2}(s)\right), \quad s \in \Gamma,
\end{gathered}
$$

where

$$
\begin{gathered}
Y_{11}(s, \sigma)=\left(\frac{1}{\pi}\left(\frac{\sin \varphi_{0}(x(s), y(\sigma))}{|x(s)-y(\sigma)|}-\frac{1}{\sigma-s}\right)-\frac{i}{2} \frac{\partial V_{0}(x(s), \sigma)}{\partial \mathbf{n}_{x}}\right. \\
\left.-\frac{i}{4}\left(\beta_{1}(s)-\beta_{2}(s)\right) V(x(s), y(\sigma))\right), \\
Y_{12}(s, \sigma)=-\frac{i}{2} \frac{\partial \mathcal{H}_{0}^{(1)}(k|x(s)-y(\sigma)|)}{\partial \mathbf{n}_{x}}, \quad Y\left[\mu_{2}\right](s)=\int_{\Gamma} \mu_{2}(\sigma) A_{22}^{-}(s, \sigma) d \sigma .
\end{gathered}
$$

It follows from Lemmas 3 and 4 in [2] that $Y_{12}(s, \sigma) \in C^{0, \lambda}(\Gamma \times \Gamma), Y_{11}(s, \sigma) \in C^{0, p}(\Gamma \times \Gamma)$, where $p=\lambda$ if $0<\lambda<1$, and $p=1-\epsilon_{0}$ for any $\epsilon_{0} \in(0,1)$ if $\lambda=1$.

Equation (13) on $\Gamma$ can be written in the form

$$
\begin{gathered}
\mu_{2}(s)+\int_{\Gamma} \mu_{1}(\sigma) A_{21}(s, \sigma) d \sigma+\int_{\Gamma} \mu_{2}(\sigma) A_{22}^{+}(s, \sigma) d \sigma \\
+\frac{1}{2}\left(\beta_{1}(s)-\beta_{2}(s)\right) \rho\left[\mu_{1}\right](s)=f_{1}(s)-f_{2}(s), \quad s \in \Gamma .
\end{gathered}
$$

Here

$$
A_{21}(s, \sigma)=\frac{i}{4}\left(\beta_{1}(s)+\beta_{2}(s)\right) V(x(s), \sigma) \in C^{0, \lambda}(\Gamma \times \Gamma)
$$

(see Lemma 4 in [2]). The properties of the integral operator with the kernel similar to $A_{22}^{ \pm}(s, \sigma)$ describe

Lemma 2. Let $\Gamma \in C^{2, \lambda}, \beta(s) \in C^{0, \lambda}(\Gamma), \lambda \in(0,1]$, and $\mathcal{A}[\nu](s)=\int_{\Gamma} \nu(\sigma) A(s, \sigma) d \sigma$, where $A(s, \sigma)=\frac{i}{4} \beta(s) \mathcal{H}_{0}^{(1)}(k|x(s)-y(\sigma)|)$. Then

1) the operator $\mathcal{A}[\nu](s)$ is compact when it acts from $C^{0}(\Gamma)$ into $C^{0}(\Gamma)$,

2) $\mathcal{A}[\nu](s) \in C^{0, \lambda / 4}(\Gamma)$ for any density $\nu(s) \in C^{0}(\Gamma)$, and $\mathcal{A}[\nu](s)$ is a bounded operator acting from $C^{0}(\Gamma)$ into $C^{0, \lambda / 4}(\Gamma)$.

Proof. The representation

$$
\begin{gathered}
A(s, \sigma)=\beta(s)\left(-\frac{1}{2 \pi} \ln |x(s)-y(\sigma)|+\frac{i}{4} h(k|x(s)-y(\sigma)|)\right) \\
=\beta(s)\left(-\frac{1}{2 \pi} \frac{I(s, \sigma)}{|x(s)-y(\sigma)|^{\lambda / 3}}+\frac{i}{4} h(k|x(s)-y(\sigma)|)\right)
\end{gathered}
$$


P. A. KRUTITSKII

holds, where $I(s, \sigma)=|x(s)-y(\sigma)|^{\lambda / 3} \ln |x(s)-y(\sigma)|$. It follows from [9, section 6.2] that $I(s, \sigma) \in C^{0, \lambda / 4}(\Gamma \times \Gamma)$. From the expansion of the function $\mathcal{H}_{0}^{(1)}(z)$ into the series [12. p. 333], we obtain: $h(z) \in C^{1}[0, \infty)$. According to the corollary to Lemma 1 in [1]: $|x(s)-y(\sigma)| \in C^{0,1}(\Gamma \times \Gamma)$, hence $h(k|x(s)-y(\sigma)|) \in C^{0,1}(\Gamma \times \Gamma)$. On the basis of (17a), the operator $\mathcal{A}[\nu](s)$ is represented as a sum of two integral operators. One of them is an integral operator with a polar kernel, while another one is an integral operator with continuous kernel. Each of these operators is compact when acting from $C^{0}(\Gamma)$ into $C^{0}(\Gamma)$. The operator with continuous kernel is compact by the Arzela-Ascoli theorem [14. p. 104], and the operator with a polar kernel is compact by virtue of [15, chapter 7, section 4]. Therefore the operator $\mathcal{A}[\nu](s)$ is compact when it acts from $C^{0}(\Gamma)$ into $C^{0}(\Gamma)$ since it is represented in the form of a sum of two compact operators. Point 1) of the lemma is proved. Let us turn to the proof of point 2).

Set $J(s, \sigma)=\beta(s) I(s, \sigma)$; then $J(s, \sigma) \in C^{0, \lambda / 4}(\Gamma \times \Gamma)$. Consider

$$
\begin{gathered}
\left|\mathcal{A}[\nu]\left(s_{2}\right)-\mathcal{A}[\nu]\left(s_{1}\right)\right| \\
\leq\|\nu\|_{C^{0}(\Gamma)}\left(\frac{1}{2 \pi} \int_{\Gamma}\left|\frac{J\left(s_{2}, \sigma\right)}{\left|x\left(s_{2}\right)-y(\sigma)\right|^{\lambda / 3}}-\frac{J\left(s_{1}, \sigma\right)}{\left|x\left(s_{1}\right)-y(\sigma)\right|^{\lambda / 3}}\right| d \sigma\right. \\
\left.+\frac{1}{4} \int_{\Gamma}\left|h\left(k\left|x\left(s_{2}\right)-y(\sigma)\right|\right)-h\left(k\left|x\left(s_{1}\right)-y(\sigma)\right|\right)\right| d \sigma\right) .
\end{gathered}
$$

For the second integral the inequality

$$
\frac{1}{4} \int_{\Gamma}\left|h\left(k\left|x\left(s_{2}\right)-y(\sigma)\right|\right)-h\left(k\left|x\left(s_{1}\right)-y(\sigma)\right|\right)\right| d \sigma \leq c_{1}\left|s_{2}-s_{1}\right|
$$

holds, where $c_{1}$ is a constant, while the first integral can be estimated in the following way:

$$
\begin{gathered}
\int_{\Gamma}\left|\frac{J\left(s_{2}, \sigma\right)}{\left|x\left(s_{2}\right)-y(\sigma)\right|^{\lambda / 3}}-\frac{J\left(s_{1}, \sigma\right)}{\left|x\left(s_{1}\right)-y(\sigma)\right|^{\lambda / 3}}\right| d \sigma \\
\leq \int_{\Gamma} \mid \frac{\left(J\left(s_{2}, \sigma\right)-J\left(s_{1}, \sigma\right)\right)\left|x\left(s_{1}\right)-y(\sigma)\right|^{\lambda / 3}}{\left|x\left(s_{2}\right)-y(\sigma)\right|^{\lambda / 3}\left|x\left(s_{1}\right)-y(\sigma)\right|^{\lambda / 3}} \\
-\frac{J\left(s_{1}, \sigma\right)\left(\left|x\left(s_{2}\right)-y(\sigma)\right|^{\lambda / 3}-\left|x\left(s_{1}\right)-y(\sigma)\right|^{\lambda / 3}\right)}{\left|x\left(s_{2}\right)-y(\sigma)\right|^{\lambda / 3}\left|x\left(s_{1}\right)-y(\sigma)\right|^{\lambda / 3}} \mid d \sigma \\
\leq \int_{\Gamma} \frac{\left|J\left(s_{2}, \sigma\right)-J\left(s_{1}, \sigma\right)\right| \cdot\left|x\left(s_{1}\right)-y(\sigma)\right|^{\lambda / 3}}{\left|x\left(s_{2}\right)-y(\sigma)\right|^{\lambda / 3}\left|x\left(s_{1}\right)-y(\sigma)\right|^{\lambda / 3}} d \sigma \\
+\int_{\Gamma} \frac{\left|J\left(s_{1}, \sigma\right)\right| \cdot|| x\left(s_{2}\right)-\left.y(\sigma)\right|^{\lambda / 3}-\left|x\left(s_{1}\right)-y(\sigma)\right|^{\lambda / 3} \mid}{\left|x\left(s_{2}\right)-y(\sigma)\right|^{\lambda / 3}\left|x\left(s_{1}\right)-y(\sigma)\right|^{\lambda / 3}} d \sigma \\
\leq c_{0}\left|s_{2}-s_{1}\right|^{\lambda / 4} \int_{\Gamma} \frac{\left(F\left(s_{1}, \sigma\right) F\left(s_{2}, \sigma\right)\right)^{\lambda / 3}}{\left|s_{2}-\sigma\right|^{\lambda / 3}\left|s_{1}-\sigma\right|^{\lambda / 3}} d \sigma,
\end{gathered}
$$


where $c_{0}$ is a constant and $F(s, \sigma)=\frac{|s-\sigma|}{|x(s)-y(\sigma)|}$. The properties of Hölder functions [9, sections 3.1, 5] are used in the derivation of the latter inequality. According to the [1, Lemma 1], $F(s, \sigma) \in C^{1}(\Gamma \times \Gamma)$, so $F(s, \sigma) \leq c_{1}$ for all $s, \sigma \in \Gamma$, where $c_{1}$ is a constant. Using the Cauchy-Bunyakovskii inequality, we obtain

$$
\begin{aligned}
& \int_{\Gamma} \frac{\left(F\left(s_{1}, \sigma\right) F\left(s_{2}, \sigma\right)\right)^{\lambda / 3}}{\left|s_{2}-\sigma\right|^{\lambda / 3}\left|s_{1}-\sigma\right|^{\lambda / 3}} d \sigma \leq c_{1}^{2 \lambda / 3} \int_{\Gamma} \frac{d \sigma}{\left|s_{2}-\sigma\right|^{\lambda / 3}\left|s_{1}-\sigma\right|^{\lambda / 3}} \\
& \leq c_{1}^{2 \lambda / 3}\left(\int_{\Gamma} \frac{d \sigma}{\left|s_{2}-\sigma\right|^{2 \lambda / 3}}\right)^{1 / 2}\left(\int_{\Gamma} \frac{d \sigma}{\left|s_{1}-\sigma\right|^{2 \lambda / 3}}\right)^{1 / 2} \leq \text { const }
\end{aligned}
$$

for any $s_{1}, s_{2} \in \Gamma$. Hence

$$
\left|\mathcal{A}[\nu]\left(s_{2}\right)-\mathcal{A}[\nu]\left(s_{1}\right)\right| \leq c\|\nu\|_{C^{0}(\Gamma)}\left|s_{2}-s_{1}\right|^{\lambda / 4}
$$

where $c$ is a constant. Therefore $\mathcal{A}[\nu](s) \in C^{0, \lambda / 4}(\Gamma)$ for any density $\nu(s) \in C^{0}(\Gamma)$. Similarly,

$$
|\mathcal{A}[\nu](s)| \leq\|\nu\|_{C^{0}(\Gamma)}\left(\mathcal{C}_{1} \int_{\Gamma} \frac{(F(s, \sigma))^{\lambda / 3}}{|s-\sigma|^{\lambda / 3}} d \sigma+\mathcal{C}_{2}\right) \leq\|\nu\|_{C^{0}(\Gamma)} c_{2}
$$

where $\mathcal{C}_{1}, \mathcal{C}_{2}, \quad c_{2}$ are constants and the inequality holds for all $s \in \Gamma$. It follows from $(17 \mathrm{~b})$ and $(17 \mathrm{c})$ that $\mathcal{A}[\nu](s)$ is a bounded operator acting from $C^{0}(\Gamma)$ into $C^{0, \lambda / 4}(\Gamma)$. The lemma is proved.

It follows from Lemma 2 that $Y\left[\mu_{2}\right](s) \in C^{0, \lambda / 4}(\Gamma)$, if $\mu_{2}(s) \in C^{0}(\Gamma)$.

Thus, if $\left\{\mu_{1}(s), \mu_{2}(s)\right\}$ is a solution to the system of equations $(15),(8)$, and $\mu_{1}(s) \in$ $C_{q}^{\omega}(\Gamma), \omega \in(0,1], \quad q \in[0,1), \mu_{2}(s) \in C^{0, \lambda / 4}(\Gamma)$, then the potential (11) satisfies all conditions of Problem $\mathbf{U}$. We arrive at

Theorem 2. Let $\Gamma \in C^{2, \lambda}$ and $\beta_{1}(s), \beta_{2}(s), f_{1}(s), f_{2}(s) \in C^{0, \lambda}(\Gamma), \lambda \in(0,1]$. If the system of equations (15), (8) has a solution $\left\{\mu_{1}(s), \mu_{2}(s)\right\}$, such that $\mu_{1}(s) \in C_{q}^{\omega}(\Gamma)$, $\omega \in(0,1], q \in[0,1), \quad \mu_{2}(s) \in C^{0, \lambda / 4}(\Gamma)$, then the solution to Problem $\mathbf{U}$ exists and is given by the formula (11).

Equation (15a) is a singular Cauchy integral equation [9, p. 315]. The goal of our further analysis is to prove solvability of the system (15), (8). In addition, we shall reduce the system (15), (8) to the uniquely solvable Fredholm integral equation of the second kind and index zero in the appropriate Banach space.

4. Regularization of the singular integral equation. Existence theorem. Inverting the Cauchy singular operator in (15a), i.e. carrying out the regularization of the singular integral equation in accordance with [9, section 99], we obtain the following 
integral equation:

$$
\begin{aligned}
\mu_{1}(s) & +\frac{1}{\mathcal{Q}_{1 / 2}(s)}\left(\int_{\Gamma} \mu_{1}(\sigma) A_{01}(s, \sigma) d \sigma+B_{0}\left[\rho\left[\mu_{1}\right]\right](s)+S_{0}\left[Y\left[\mu_{2}\right]\right](s)\right. \\
& \left.+\int_{\Gamma} \mu_{2}(\sigma) A_{02}(s, \sigma) d \sigma+\sum_{n=0}^{N-1} G_{n} s^{n}\right)=\frac{\Phi_{0}(s)}{\mathcal{Q}_{1 / 2}(s)}, \quad s \in \Gamma,
\end{aligned}
$$

where $G_{0}, \ldots, G_{N-1}$ are arbitrary constants; $\mathcal{Q}_{1 / 2}(s)$ is defined in (14a);

$$
\begin{gathered}
A_{0 j}(s, \sigma)=-\frac{1}{\pi} \int_{\Gamma} \frac{Y_{1 j}(\xi, \sigma) \mathcal{Q}_{1 / 2}(\xi)}{\xi-s} d \xi, \quad j=1,2 ; \\
S_{0}\left[Y\left[\mu_{2}\right]\right](s)=-\frac{1}{\pi} \int_{\Gamma} \frac{\mathcal{Q}_{1 / 2}(\xi) Y\left[\mu_{2}\right](\xi)}{\xi-s} d \xi ; \\
B_{0}\left[\rho\left[\mu_{1}\right]\right](s)=\frac{1}{2 \pi} \int_{\Gamma} \frac{\mathcal{Q}_{1 / 2}(\xi)\left(\beta_{1}(\xi)+\beta_{2}(\xi)\right) \rho\left[\mu_{1}\right](\xi)}{\xi-s} d \xi ; \\
\Phi_{0}(s)=\frac{1}{\pi} \int_{\Gamma} \frac{\mathcal{Q}_{1 / 2}(\xi)\left(f_{1}(\xi)+f_{2}(\xi)\right)}{\xi-s} d \xi .
\end{gathered}
$$

Let $\omega \in(0,1], \quad q \in[0,1)$. Note that if the functions $\mu_{1}(s) \in C_{q}^{\omega}(\Gamma)$ and $\mu_{2}(s) \in$ $C^{0, \lambda / 4}(\Gamma)$ obey equation (18) for some constants $G_{0}, \ldots, G_{N-1}$, then they obey equation (15a). (This fact can be easily shown by acting with the singular integral operator with the kernel $(\sigma-s)^{-1}$ on equation (18), which transforms into equation (15a).) Let us study properties of the functions defined in (19). Obviously, $\mathcal{Q}_{1 / 2}(s) \in C^{0,1 / 2}(\Gamma)$. If $\mu_{1}(s) \in C_{q}^{\omega}(\Gamma)$, then it follows from statement (b) of Lemma 1 that $\rho\left[\mu_{1}\right](s) \in C^{0,1-q}(\Gamma)$. Using the properties of singular integrals [9, section 18], we conclude that $B_{0}\left[\rho\left[\mu_{1}\right]\right](s)$, $S_{0}\left[Y\left[\mu_{2}\right]\right](s), \Phi_{0}(s)$ are Hölder continuous functions on $\Gamma$, and $A_{0 j}(s, \sigma)$ is a Hölder continuous function on $\Gamma$ in both variables for $j=1,2$. Moreover, $B_{0}\left[\rho\left[\mu_{1}\right]\right](s) \in C^{0, \varsigma}(\Gamma)$, where $\varsigma=\min \{1 / 2,1-q, \lambda\} ; \quad S_{0}\left[Y\left[\mu_{2}\right]\right](s) \in C^{0, \lambda / 4}(\Gamma) ; \quad \Phi_{0}(s) \in C^{0, \eta}(\Gamma)$, where $\eta=\min \{1 / 2, \lambda\}$; and $A_{0 j}(s, \sigma)$ belongs to the class $C^{0, \eta}(\Gamma)$ in $s$ uniformly with respect to $\sigma \in \Gamma$ for $j=1,2$. Therefore, if functions $\mu_{1}(s) \in C_{q}^{\omega}(\Gamma), \mu_{2}(s) \in C^{0, \lambda / 4}(\Gamma)$ (here $\omega \in(0,1], q \in[0,1))$ satisfy equation $(18)$, then $\mu_{1}(s)$ belongs to $C_{1 / 2}^{\lambda / 4}(\Gamma)$ automatically, i.e. $q=1 / 2$ and $\omega=\min \{\varsigma, \eta, \lambda / 4\}=\lambda / 4$. Thus, further on we shall look for $\mu_{1}(s)$ in the space $C_{1 / 2}^{\lambda / 4}(\Gamma)$.

Let us choose the constants $G_{0}, \ldots, G_{N-1}$ in order to satisfy conditions (8). Substituting $\mu_{1}(s)$ from (18) into (8), we obtain the linear system of equations for determination of $G_{0}, \ldots, G_{N-1}$

$$
\begin{gathered}
\int_{\Gamma} \mu_{1}(\sigma) l_{n}^{(1)}(\sigma) d \sigma+\int_{\Gamma} \mu_{2}(\sigma) l_{n}^{(2)}(\sigma) d \sigma+\int_{\Gamma} \frac{1}{2}\left(\beta_{1}(\xi)+\beta_{2}(\xi)\right) \rho\left[\mu_{1}\right](\xi) g_{n}(\xi) d \xi \\
-\int_{\Gamma} Y\left[\mu_{2}\right](\xi) g_{n}(\xi) d \xi+\sum_{m=0}^{N-1} W_{n m} G_{m}=H_{n}, \quad n=1, \ldots, N
\end{gathered}
$$


where

$$
\begin{gathered}
g_{n}(\xi)=\frac{\mathcal{Q}_{1 / 2}(\xi)}{\pi} \int_{a_{n}}^{b_{n}} \frac{1}{\mathcal{Q}_{1 / 2}(s)} \frac{1}{\xi-s} d s, \quad \xi \in \Gamma ; \\
l_{n}^{(j)}(\sigma)=-\int_{\Gamma} Y_{1 j}(\xi, \sigma) g_{n}(\xi) d \xi, \quad j=1,2, \quad \sigma \in \Gamma ; \\
H_{n}=\int_{\Gamma}\left(f_{1}(\xi)+f_{2}(\xi)\right) g_{n}(\xi) d \xi ; \quad W_{n m}=\int_{a_{n}}^{b_{n}} \mathcal{Q}_{1 / 2}^{-1}(s) s^{m} d s, \quad m=0, \ldots, N-1 .
\end{gathered}
$$

Here $W_{n m}$ are the elements of the matrix $W$ with dimensions $N \times N$. As is shown in [2, lemma 7], the inverse matrix $W^{-1}$ for $W$ exists and has the elements $\left(W^{-1}\right)_{n m}$, $n=0, \ldots, N-1, \quad m=1, \ldots, N$. Inverting the matrix $W$ in (20), we express the constants $G_{0}, \ldots, G_{N-1}$ in terms of $\mu_{1}(s)$ :

$$
\begin{aligned}
G_{n} & =\sum_{m=1}^{N}\left(W^{-1}\right)_{n m}\left\{H_{m}-\int_{\Gamma} \mu_{1}(\sigma) l_{m}^{(1)}(\sigma) d \sigma-\int_{\Gamma} \mu_{2}(\sigma) l_{m}^{(2)}(\sigma) d \sigma\right. \\
& \left.+\int_{\Gamma} Y\left[\mu_{2}\right](\xi) g_{m}(\xi) d \xi-\int_{\Gamma} \frac{1}{2}\left(\beta_{1}(\xi)+\beta_{2}(\xi)\right) \rho\left[\mu_{1}\right](\xi) g_{m}(\xi) d \xi\right\} .
\end{aligned}
$$

REMARK. It follows from the formulas for the integrals calculated in [13, p. 910] that the coefficients defined in (21) satisfy the identities:

$$
\begin{gathered}
\sum_{n=1}^{N} g_{n}(\xi) \equiv 0, \quad \sum_{n=1}^{N} l_{n}^{(j)}(\sigma) \equiv 0, \quad \xi, \sigma \in \bigcup_{n=1}^{N}\left(a_{n}, b_{n}\right), \quad j=1,2 ; \\
\sum_{n=1}^{N} H_{n}=0 ; \quad \sum_{n=1}^{N} W_{n m}=\int_{\Gamma} \mathcal{Q}_{1 / 2}^{-1}(s) s^{m} d s= \begin{cases}0, & m=0, \ldots, N-2 ; \\
\pi, & m=N-1 .\end{cases}
\end{gathered}
$$

Therefore, if the number of slits is $N=1$, then the solution to the equation (20) is $G_{0}=0$.

Substituting the constants $G_{n}$ from (22) into equation (18), we obtain the integral equation for $\mu_{1}(s), \mu_{2}(s)$ :

$$
\begin{gathered}
\mu_{1}(s)+\frac{1}{\mathcal{Q}_{1 / 2}(s)}\left(\int_{\Gamma} \mu_{1}(\sigma) A_{11}(s, \sigma) d \sigma+\int_{\Gamma} \mu_{2}(\sigma) A_{12}(s, \sigma) d \sigma+S\left[Y\left[\mu_{2}\right]\right](s)\right) \\
+\frac{B\left[\rho\left[\mu_{1}\right]\right](s)}{\mathcal{Q}_{1 / 2}(s)}=\frac{\Phi(s)}{\mathcal{Q}_{1 / 2}(s)}, \quad s \in \Gamma,
\end{gathered}
$$


where

$$
\begin{gathered}
A_{1 j}(s, \sigma)=A_{0 j}(s, \sigma)-\sum_{n=0}^{N-1} s^{n} \sum_{m=1}^{N}\left(W^{-1}\right)_{n m} l_{m}^{(j)}(\sigma), \quad j=1,2, \\
\Phi(s)=\Phi_{0}(s)-\sum_{n=0}^{N-1} s^{n} \sum_{m=1}^{N}\left(W^{-1}\right)_{n m} H_{m}, \\
S\left[Y\left[\mu_{2}\right]\right](s)=S_{0}\left[Y\left[\mu_{2}\right]\right](s)+\sum_{n=0}^{N-1} s^{n} \int_{\Gamma} Y\left[\mu_{2}\right](\xi) \sum_{m=1}^{N}\left(W^{-1}\right)_{n m} g_{m}(\xi) d \xi \\
B\left[\rho\left[\mu_{1}\right]\right](s)=B_{0}\left[\rho\left[\mu_{1}\right]\right](s) \\
-\sum_{n=0}^{N-1} s^{n} \int_{\Gamma} \frac{1}{2}\left(\beta_{1}(\xi)+\beta_{2}(\xi)\right) \rho\left[\mu_{1}\right](\xi) \sum_{m=1}^{N}\left(W^{-1}\right)_{n m} g_{m}(\xi) d \xi .
\end{gathered}
$$

If $\left\{\mu_{1}(s), \mu_{2}(s)\right\}$ is an arbitrary solution to equation (23), such that $\mu_{1}(s) \in C_{1 / 2}^{\lambda / 4}(\Gamma)$, $\mu_{2}(s) \in C^{0, \lambda / 4}(\Gamma)$, then $\mu_{1}(s)$ satisfies conditions (8) automatically (this fact can be shown by direct verification).

It follows from properties of the functions $A_{0 j}(s, \sigma), B_{0}\left[\rho\left[\mu_{1}\right]\right](s), \Phi_{0}(s)$, defined in (19), and from formulas (24), that $\Phi(s) \in C^{0, \eta}(\Gamma)$, where $\eta=\min \{1 / 2, \lambda\}$; the function $A_{1 j}(s, \sigma)$ with $j=1,2$ is Hölder continuous in both variables and belongs to the class $C^{0, \eta}(\Gamma)$ in $s$ uniformly with respect to $\sigma \in \Gamma$; if $\mu_{2}(s) \in C^{0}(\Gamma)$, then $S\left[Y\left[\mu_{2}\right]\right](s) \in$ $C^{0, \lambda / 4}(\Gamma)$; if $\mu_{1}(s) \in C_{1 / 2}^{\lambda / 4}(\Gamma)$, then $\rho\left[\mu_{1}\right](s) \in C^{0,1 / 2}(\Gamma)$ and $B\left[\rho\left[\mu_{1}\right]\right](s) \in C^{0, \eta}(\Gamma)$. Let us introduce the new unknown function $\mu_{1 *}(s)=\mu_{1}(s) \mathcal{Q}_{1 / 2}(s) \in C^{0, \lambda / 4}(\Gamma)$ instead of $\mu_{1}(s) \in C_{1 / 2}^{\lambda / 4}(\Gamma)$ and rewrite equation (23) in the form

$$
\mu_{1 *}(s)+\mathcal{A}_{11}\left[\mu_{1 *}\right](s)+\mathcal{B}\left[\mu_{1 *}\right](s)+\mathcal{A}_{12}\left[\mu_{2}\right](s)+\mathcal{S}\left[\mu_{2}\right](s)=\Phi(s), \quad s \in \Gamma,
$$

where, taking into account (14b),

$$
\begin{array}{cc}
\mathcal{A}_{11}\left[\mu_{1 *}\right](s)=\int_{\Gamma} \mu_{1 *}(\sigma) \mathcal{Q}_{1 / 2}^{-1}(\sigma) A_{11}(s, \sigma) d \sigma, & \mathcal{B}\left[\mu_{1 *}\right](s)=B\left[\rho_{1 / 2}\left[\mu_{1 *}\right]\right](s), \\
\mathcal{A}_{12}\left[\mu_{2}\right](s)=\int_{\Gamma} \mu_{2}(\sigma) A_{12}(s, \sigma) d \sigma, & \mathcal{S}\left[\mu_{2}\right](s)=S\left[Y\left[\mu_{2}\right]\right](s) .
\end{array}
$$

If $\mu_{1 *}(s) \in C^{0}(\Gamma)$, then $\mathcal{A}_{11}\left[\mu_{1 *}\right](s) \in C^{0, \eta}(\Gamma)$, and according to Lemma $1(\mathrm{a}): \rho_{1 / 2}\left[\mu_{1 *}\right](s)$ $\in C^{0,1 / 2}(\Gamma)$, whence $\mathcal{B}\left[\mu_{1 *}\right](s) \in C^{0, \eta}(\Gamma)$. From the above arguments we obtain

Lemma 3. Let $\Gamma \in C^{2, \lambda}, \beta_{1}(s), \beta_{2}(s) \in C^{0, \lambda}(\Gamma), \lambda \in(0,1]$ and $\eta=\min \{1 / 2, \lambda\}$. If functions $\mu_{1 *}(s), \quad \mu_{2}(s)$ from $C^{0}(\Gamma)$ obey equation $(25)$, where $\Phi(s) \in C^{0, \eta}(\Gamma)$, then $\mu_{1 *}(s)$ belongs to $C^{0, \lambda / 4}(\Gamma)$.

In terms of the function $\mu_{1 *}(s)$, we rewrite equation (15b) in the form

$$
\mu_{2}(s)+\mathcal{A}_{21}\left[\mu_{1 *}\right]+\mathcal{E}\left[\mu_{1 *}\right](s)+\mathcal{A}_{22}\left[\mu_{2}\right](s)=f_{1}(s)-f_{2}(s), \quad s \in \Gamma,
$$


where, taking into account (14b): $\quad \mathcal{E}\left[\mu_{1 *}\right](s)=\frac{1}{2}\left(\beta_{1}(s)-\beta_{2}(s)\right) \rho_{1 / 2}\left[\mu_{1 *}\right](s) \quad$ and

$$
\mathcal{A}_{22}\left[\mu_{2}\right](s)=\int_{\Gamma} \mu_{2}(\sigma) A_{22}^{+}(s, \sigma) d \sigma, \quad \mathcal{A}_{21}\left[\mu_{1 *}\right](s)=\int_{\Gamma} \mu_{1 *}(\sigma) \mathcal{Q}_{1 / 2}^{-1}(\sigma) A_{21}(s, \sigma) d \sigma .
$$

It follows from (16) that $\mathcal{A}_{21}\left[\mu_{1 *}\right](s) \in C^{0, \lambda}(\Gamma)$ for any $\mu_{1 *}(s) \in C^{0}(\Gamma)$. By Lemma 1 (a) we obtain that $\rho_{1 / 2}\left[\mu_{1 *}\right](s) \in C^{0,1 / 2}(\Gamma)$ for any $\mu_{1 *}(s) \in C^{0}(\Gamma)$. It follows from statement 2 ) of Lemma 2 that $\mathcal{A}_{22}\left[\mu_{2}\right](s) \in C^{0, \lambda / 4}(\Gamma)$ for any $\mu_{2}(s) \in C^{0}(\Gamma)$. Hence we arrive at

Lemma 4. Let $\Gamma \in C^{2, \lambda}, \quad \beta_{1}(s), \beta_{2}(s), f_{1}(s), f_{2}(s) \in C^{0, \lambda}(\Gamma) ; \lambda \in(0,1]$. If functions $\mu_{1 *}(s), \mu_{2}(s)$ belong to $C^{0}(\Gamma)$ and obey equation $(26)$, then $\mu_{2}(s) \in C^{0, \lambda / 4}(\Gamma)$.

On the basis of Lemmas 3, 4, we will look for a solution $\left\{\mu_{1 *}(s), \mu_{2}(s)\right\}$ to the system (25), (26) among functions $\mu_{1 *}(s), \mu_{2}(s) \in C^{0}(\Gamma)$. Consider the operators in equation (26). Note that $A_{21}(s, \sigma) \in C^{0}(\Gamma \times \Gamma)$ according to (16). Therefore, it can be verified directly using the Arzela-Ascoli theorem [14, p. 104] that $\mathcal{A}_{21}\left[\mu_{1 *}\right](s)$ is a compact operator acting from $C^{0}(\Gamma)$ into $C^{0}(\Gamma)$. In view of statement 1 ) of Lemma 2 , the operator $\mathcal{A}_{22}\left[\mu_{2}\right](s)$ is compact when acting from $C^{0}(\Gamma)$ into $C^{0}(\Gamma)$.

By Lemma $1(\mathrm{a}), \rho_{1 / 2}\left[\mu_{1 *}\right](s)$ is a bounded operator acting from $C^{0}(\Gamma)$ into $C^{0,1 / 2}(\Gamma)$. According to [16, p. 915], the space $C^{0,1 / 2}(\Gamma)$ is compactly embedded into the space $C^{0,1 / 4}(\Gamma)$. Therefore the operator $\rho_{1 / 2}\left[\mu_{1 *}\right](s)$ is compact when acting from $C^{0}(\Gamma)$ into $C^{0,1 / 4}(\Gamma)$, since it is represented as a composition of a bounded operator acting from $C^{0}(\Gamma)$ into $C^{0,1 / 2}(\Gamma)$, and a compact operator embedding $C^{0,1 / 2}(\Gamma)$ into $C^{0,1 / 4}(\Gamma)$. Obviously, the operator embedding the space $C^{0,1 / 4}(\Gamma)$ into $C^{0}(\Gamma)$ is bounded. Therefore the operator $\rho_{1 / 2}\left[\mu_{1 *}\right](s)$ is compact when acting from $C^{0}(\Gamma)$ into $C^{0}(\Gamma)$, since it is represented as a composition of a compact operator acting from $C^{0}(\Gamma)$ into $C^{0,1 / 4}(\Gamma)$ and a bounded operator embedding $C^{0,1 / 4}(\Gamma)$ into $C^{0}(\Gamma)$. Hence the operator $\mathcal{E}\left[\mu_{1 *}\right](s)$ is compact from $C^{0}(\Gamma)$ into $C^{0}(\Gamma)$ as well.

Let us study the operators in equation (25). As mentioned above,

$$
A_{1 j}(s, \sigma) \in C^{0}(\Gamma \times \Gamma)
$$

for $j=1,2$. Therefore, one can prove using the Arzela-Ascoli theorem [14, p. 104] that $\mathcal{A}_{11}\left[\mu_{1 *}\right](s)$ and $\mathcal{A}_{12}\left[\mu_{2}\right](s)$ are compact operators acting from $C^{0}(\Gamma)$ into $C^{0}(\Gamma)$. Let us prove

Lemma 5. Let $\Gamma \in C^{2, \lambda}, \quad \beta(s) \in C^{0, \lambda}(\Gamma), \quad \lambda \in(0,1] ; \nu(s) \in C^{0, \gamma}(\Gamma)$, where $\gamma$ is a fixed number from the interval $(0,1]$, and $D[\nu](s)=\int_{\Gamma} \frac{\mathcal{Q}_{1 / 2}(\sigma) \beta(\sigma) \nu(\sigma)}{\sigma-s} d \sigma$. Then the operator $D[\nu](s)$ is bounded as an operator acting from $C^{0, \gamma}(\Gamma)$ into $C^{0}(\Gamma)$.

Proof. We represent the operator $D[\nu](s)$ in the form

$$
D[\nu](s)=\frac{1}{\pi} \int_{\Gamma} \frac{\mathcal{Q}_{1 / 2}(\sigma) \beta(\sigma)(\nu(\sigma)-\nu(s))}{\sigma-s} d \sigma+\nu(s) b(s),
$$


where $b(s)=\frac{1}{\pi} \int_{\Gamma} \frac{\mathcal{Q}_{1 / 2}(\sigma) \beta(\sigma)}{\sigma-s} d \sigma$. It follows from properties of singular integrals 9 , section 18] that $b(s) \in C^{0, \eta}(\Gamma)$ since the function $\mathcal{Q}_{1 / 2}(\sigma) \beta(\sigma)$ belongs to the class $C^{0, \eta}(\Gamma)$ with $\eta=\min \{1 / 2, \lambda\}$ and equals zero at each end point of the contour $\Gamma$. Estimating the norm of $D[\nu](s)$ in $C^{0}(\Gamma)$, where $\nu(s) \in C^{0, \gamma}(\Gamma)$, we obtain

$$
\begin{aligned}
\|D[\nu](\cdot)\|_{C^{0}(\Gamma)} \leq & \frac{1}{\pi}\left(\left\|\int_{\Gamma}\left|\mathcal{Q}_{1 / 2}(\sigma) \beta(\sigma)\right||\sigma-\cdot|^{\gamma-1} d \sigma\right\|_{C^{0}(\Gamma)}\|\nu\|_{C^{0, \gamma}(\Gamma)}\right. \\
& \left.+\|b\|_{C^{0}(\Gamma)}\|\nu\|_{C^{0}(\Gamma)}\right) \leq \mathrm{const}\|\nu\|_{C^{0, \gamma}(\Gamma)} .
\end{aligned}
$$

Therefore $D[\nu](s)$ is a bounded operator acting from $C^{0, \gamma}(\Gamma)$ into $C^{0}(\Gamma)$. The lemma is proved.

Now we will prove that the operator $\mathcal{B}\left[\mu_{1 *}\right](s)$ is compact from $C^{0}(\Gamma)$ into $C^{0}(\Gamma)$. Consider the operator $B_{0}\left[\rho_{1 / 2}\right](s)$ defined in (19) and acting on the function $\rho_{1 / 2}(\sigma)$ from the space $C^{0,1 / 4}(\Gamma)$. By Lemma 5 , the operator $B_{0}\left[\rho_{1 / 2}\right](s)$ is bounded when acting from $C^{0,1 / 4}(\Gamma)$ into $C^{0}(\Gamma)$. The operator $B\left[\rho_{1 / 2}\right](s)$ is bounded from $C^{0,1 / 4}(\Gamma)$ into $C^{0}(\Gamma)$ since it is represented in $(24)$ in the form of a sum of the operator $B_{0}\left[\rho_{1 / 2}\right](s)$ and a finite-dimensional, so bounded, operator. It follows from boundedness of the operator $B\left[\rho_{1 / 2}\right](s)$ from $C^{0,1 / 4}(\Gamma)$ into $C^{0}(\Gamma)$ and from compactness of the operator $\rho_{1 / 2}\left[\mu_{1 *}\right](s)$ from $C^{0}(\Gamma)$ into $C^{0,1 / 4}(\Gamma)$ that $\mathcal{B}\left[\mu_{1 *}\right](s)$ is a compact operator acting from $C^{0}(\Gamma)$ into $C^{0}(\Gamma)$ since it is represented in the form of a composition of compact and bounded operators. Hence $\mathcal{A}_{11}\left[\mu_{1 *}\right](s)+\mathcal{B}\left[\mu_{1 *}\right](s)$ is a compact operator acting from $C^{0}(\Gamma)$ into $C^{0}(\Gamma)$.

Let us show that the operator $\mathcal{S}\left[\mu_{2}\right](s)$ is compact when acting from $C^{0}(\Gamma)$ into $C^{0}(\Gamma)$. Consider the operator $Y\left[\mu_{2}\right](s)$. By statement 2$)$ of Lemma 2 we obtain that $Y\left[\mu_{2}\right](s)$ is a bounded operator acting from $C^{0}(\Gamma)$ into $C^{0, \lambda / 4}(\Gamma)$. It follows from [16, p. 915] that the space $C^{0, \lambda / 4}(\Gamma)$ is compactly embedded in the space $C^{0, \lambda / 5}(\Gamma)$. The operator $Y\left[\mu_{2}\right](s)$ is compact from $C^{0}(\Gamma)$ into $C^{0, \lambda / 5}(\Gamma)$ since it is represented in the form of a composition of a bounded operator acting from $C^{0}(\Gamma)$ into $C^{0, \lambda / 4}(\Gamma)$ and a compact operator embedding $C^{0, \lambda / 4}(\Gamma)$ into $C^{0, \lambda / 5}(\Gamma)$. Consider the operator $S_{0}[Y](s)$ defined in (19) and acting on the function $Y(\sigma)$ from the space $C^{0, \lambda / 5}(\Gamma)$. Using Lemma 5 , where $\beta(s) \equiv 1$, we observe that $S_{0}[Y](s)$ is a bounded operator, acting from $C^{0, \lambda / 5}(\Gamma)$ into $C^{0}(\Gamma)$. The operator $S[Y](s)$ is bounded from $C^{0, \lambda / 5}(\Gamma)$ into $C^{0}(\Gamma)$, since it is represented in (24) in the form of a sum of the operator $S_{0}[Y](s)$ and a finite-dimensional, so bounded, operator. It follows from boundedness of the operator $S[Y](s)$ from $C^{0, \lambda / 5}(\Gamma)$ into $C^{0}(\Gamma)$ and from compactness of the operator $Y\left[\mu_{2}\right](s)$ from $C^{0}(\Gamma)$ into $C^{0, \lambda / 5}(\Gamma)$ that $\mathcal{S}\left[\mu_{2}\right](s)$ is a compact operator acting from $C^{0}(\Gamma)$ into $C^{0}(\Gamma)$ since it is represented in the form of a composition of a compact operator and a bounded operator. Hence $\mathcal{A}_{12}\left[\mu_{2}\right](s)+\mathcal{S}\left[\mu_{2}\right](s)$ is a compact operator acting from $C^{0}(\Gamma)$ into $C^{0}(\Gamma)$. 
Consider columns $\vec{\mu}=\left(\mu_{1 *}, \mu_{2}\right)^{T}, \vec{\Phi}=\left(\Phi, f_{1}-f_{2}\right)^{T}$ in the Banach space $C^{0}(\Gamma) \times C^{0}(\Gamma)$ with the norm

$$
\|\vec{\mu}\|_{C^{0}(\Gamma) \times C^{0}(\Gamma)}=\left\|\mu_{1 *}\right\|_{C^{0}(\Gamma)}+\left\|\mu_{2}\right\|_{C^{0}(\Gamma)} .
$$

We rewrite system (25), (26) in the form of one equation

$$
(\mathbf{I}+\mathbf{R}) \vec{\mu}=\vec{\Phi}, \quad \mathbf{R}=\left(\begin{array}{cc}
\mathcal{A}_{11}+\mathcal{B} & \mathcal{A}_{12}+\mathcal{S} \\
\mathcal{A}_{21}+\mathcal{E} & \mathcal{A}_{22}
\end{array}\right)
$$

where $\mathbf{I}$ is a unit operator in the space $C^{0}(\Gamma) \times C^{0}(\Gamma)$. The operator $\mathbf{R}$ is a compact operator mapping the space $C^{0}(\Gamma) \times C^{0}(\Gamma)$ into itself since all operators forming $\mathbf{R}$ are compact. Therefore (27) is a Fredholm equation of the second kind and index zero in this space [17, p. 67], [18, pp. 473-479], [19, section 20.5]. Thus we have proved

Lemma 6. If $\Gamma \in C^{2, \lambda}, \beta_{1}(s), \beta_{2}(s) \in C^{0, \lambda}(\Gamma), \lambda \in(0,1]$, then (27) is a Fredholm equation of the second kind and index zero in the space $C^{0}(\Gamma) \times C^{0}(\Gamma)$.

Now we will prove that (27) is a uniquely solvable integral equation in the space $C^{0}(\Gamma) \times C^{0}(\Gamma)$.

Lemma 7. If $\Gamma \in C^{2, \lambda}, \beta_{1}(s), \beta_{2}(s) \in C^{0, \lambda}(\Gamma)$ and $\lambda \in(0,1]$, then the Fredholm equation of the second kind and index zero (27) has a unique solution $\vec{\mu}=\left(\mu_{1 *}, \mu_{2}\right)^{T} \in$ $C^{0}(\Gamma) \times C^{0}(\Gamma)$ for any $\vec{\Phi}=\left(\Phi, f_{1}-f_{2}\right)^{T} \in C^{0}(\Gamma) \times C^{0}(\Gamma)$.

Proof. We shall show that the homogeneous equation (27) (with $\vec{\Phi}=\left(\Phi, f_{1}-f_{2}\right)^{T} \equiv$ $(0,0)^{T}$ for $\left.s \in \Gamma\right)$ has only trivial solution in $C^{0}(\Gamma) \times C^{0}(\Gamma)$. Suppose that $\vec{\mu}^{0}=$ $\left(\mu_{1 *}^{0}(s), \mu_{2}^{0}(s)\right)^{T} \in C^{0}(\Gamma) \times C^{0}(\Gamma)$ is a solution to the homogeneous equation (27). This solution converts homogeneous equations (25), (26) into identities. By Lemmas 3, 4: $\mu_{1 *}^{0}(s) \in C^{0, \lambda / 4}(\Gamma), \quad \mu_{2}^{0}(s) \in C^{0, \lambda / 4}(\Gamma)$. Then the function $\mu_{1}^{0}(s)=\mu_{1 *}^{0}(s) \mathcal{Q}_{1 / 2}^{-1}(s)$ belongs to the space $C_{1 / 2}^{\lambda / 4}(\Gamma)$. Besides, the functions $\mu_{1}^{0}(s), \mu_{2}^{0}(s)$ convert homogeneous equations (23), (15b) into identities. Using homogeneous identities (23), one can verify that the function $\mu_{1}^{0}(s)$ satisfies conditions (8). Acting on the homogeneous identity (23) with a singular integral operator with the kernel $(\sigma-s)^{-1}$, we obtain that the functions $\mu_{1}^{0}(s)$ and $\mu_{2}^{0}(s)$ obey homogeneous equation (15a). Therefore $\left\{\mu_{1}^{0}(s), \mu_{2}^{0}(s)\right\}$ is a solution to the homogeneous equations (15), (8). By Theorem 2 the function $u^{0}\left[\mu_{1}^{0}, \mu_{2}^{0}\right](x)$ defined by formula (11) is a solution to the homogeneous Problem U. By Theorem 1, we see that $u^{0}\left[\mu_{1}^{0}, \mu_{2}^{0}\right](x) \equiv 0$ for $x \in R^{2} \backslash \Gamma$. Using the limit formulas for tangent and normal derivatives of potentials [1], we obtain

$$
\begin{aligned}
& \left.\frac{\partial u^{0}\left[\mu_{1}^{0}, \mu_{2}^{0}\right](x)}{\partial \tau_{x}}\right|_{x(s) \in \Gamma^{+}}-\left.\frac{\partial u^{0}\left[\mu_{1}^{0}, \mu_{2}^{0}\right](x)}{\partial \tau_{x}}\right|_{x(s) \in \Gamma^{-}}=\mu_{1}^{0}(s) \equiv 0, \quad s \in \Gamma, \\
& \left.\frac{\partial u^{0}\left[\mu_{1}^{0}, \mu_{2}^{0}\right](x)}{\partial \mathbf{n}_{x}}\right|_{x(s) \in \Gamma^{+}}-\left.\frac{\partial u^{0}\left[\mu_{1}^{0}, \mu_{2}^{0}\right](x)}{\partial \mathbf{n}_{x}}\right|_{x(s) \in \Gamma^{-}}=\mu_{2}^{0}(s) \equiv 0, \quad s \in \Gamma .
\end{aligned}
$$

Consequently, $\quad \mu_{1 *}^{0}(s)=\mu_{1}^{0}(s) \mathcal{Q}_{1 / 2}(s) \equiv 0 \quad$ for $\quad s \in \Gamma, \quad$ so $\vec{\mu}^{0}=\left(\mu_{1 *}^{0}(s), \mu_{2}^{0}(s)\right)^{T} \equiv$ $(0,0)^{T}$ for $s \in \Gamma$. Therefore, the homogeneous Fredholm integral equation of the second kind and index zero (27) has only the trivial solution in $C^{0}(\Gamma) \times C^{0}(\Gamma)$. The statement 
of the lemma follows from Fredholm's theorem [17, p. 67], [18, pp. 473-479], [19, section 20.5].

Now we turn to the analysis of the solvability of the system (15), (8).

Let $f_{1}(s), f_{2}(s) \in C^{0, \lambda}(\Gamma)$. Then the function $\Phi(s)$, constructed with the help of formulas (19) and (24), belongs to the class $C^{0, \eta}(\Gamma)$ with $\eta=\min \{1 / 2, \lambda\}$. By Lemma 7 equation (27) with the right side $\left(\Phi(s), f_{1}(s)-f_{2}(s)\right)^{T}$ has the unique solution $\left(\mu_{1 *}(s), \mu_{2}(s)\right)^{T} \in C^{0}(\Gamma) \times C^{0}(\Gamma)$, which converts equations (25), (26) into an identity. By Lemmas 3, 4: $\mu_{1 *}(s) \in C^{0, \lambda / 4}(\Gamma), \quad \mu_{2}(s) \in C^{0, \lambda / 4}(\Gamma)$. It follows from derivation of equations (25), (26) that the functions $\mu_{1}(s)=\mu_{1 *}(s) \mathcal{Q}_{1 / 2}^{-1}(s) \in C_{1 / 2}^{\lambda / 4}(\Gamma)$ and $\mu_{2}(s) \in C^{0, \lambda / 4}(\Gamma)$ convert equations (23) and (15b) into identities. Using identity (23), one can verify that $\mu_{1}(s)$ satisfies conditions (8). Acting on the identity (23) by a singular integral operator with the kernel $(\sigma-s)^{-1}$, we obtain that $\mu_{1}(s)$ and $\mu_{2}(s)$ satisfy equation (15a). Thus we have proved the following statement.

Lemma 8. Let $\Gamma \in C^{2, \lambda} ; \beta_{1}(s), \beta_{2}(s), f_{1}(s), f_{2}(s) \in C^{0, \lambda}(\Gamma) ; \quad \lambda \in(0,1]$. Then the system of equations (15), (8) has a solution $\left\{\mu_{1}(s), \mu_{2}(s)\right\}$ such that $\mu_{2}(s) \in C^{0, \lambda / 4}(\Gamma)$, $\mu_{1}(s) \in C_{1 / 2}^{\lambda / 4}(\Gamma)$. Moreover, this solution can be written as $\left\{\mu_{1 *}(s) \mathcal{Q}_{1 / 2}^{-1}(s), \mu_{2}(s)\right\}$, where the functions $\mu_{1 *}(s) \in C^{0, \lambda / 4}(\Gamma)$ and $\mu_{2}(s) \in C^{0, \lambda / 4}(\Gamma)$ form the unique solution of equation (27) in the space $C^{0}(\Gamma) \times C^{0}(\Gamma)$, assured by Lemma 7 .

REMARK. The system of equations (15), (8) has no more than one solution $\left\{\mu_{1}(s), \mu_{2}(s)\right\}$ such that $\mu_{1}(s) \in C_{q}^{\omega}(\Gamma), \mu_{2}(s) \in C^{0, \omega_{0}}(\Gamma)$, where $\omega \in(0,1], q \in[0,1)$, $\omega_{0} \in(0,1]$. This statement can be proved using the technique in the proof of Lemma 7 . Consequently, the solution assured by Lemma 8 is unique.

The existence theorem for the Problem $\mathbf{U}$ follows from Lemma 8 and Theorem 2.

Theorem 3. Let $\Gamma \in C^{2, \lambda} ; \beta_{1}(s), \beta_{2}(s), f_{1}(s), f_{2}(s) \in C^{0, \lambda}(\Gamma) ; \lambda \in(0,1]$. A solution to Problem $\mathbf{U}$ exists and is given by the formula (11), where functions $\mu_{1}(s) \in C_{1 / 2}^{\lambda / 4}(\Gamma)$ and $\mu_{2}(s) \in C^{0, \lambda / 4}(\Gamma)$ form a solution to the system of equations (15), (8), assured by Lemma 8 .

By Theorem 1, a solution to Problem $\mathbf{U}$ obtained in Theorem 3 is unique. It follows from [1, theorem 5.3] that the solution to Problem $\mathbf{U}$ given by formula (11) satisfies condition (1) with $\varepsilon=-1 / 2$. The explicit formulas for singularities of the gradient of the solution at the ends of the slits $\Gamma$ will be presented in the next section.

5. Behavior of the gradient of a solution at the ends of the slits. Let $x(d)$ be one of the end points of the contour $\Gamma: x(d) \in X ; d=a_{n}$ or $d=b_{n}, n=1, \ldots, N$. Let us study the asymptotic behavior of the directional derivative $\frac{\partial u\left[\mu_{1}, \mu_{2}\right]}{\partial \mathbf{w}}(x)$ of a solution to Problem $\mathbf{U}$ in the direction of $\mathbf{w}=(\cos \vartheta, \sin \vartheta), \quad \vartheta \in[0,2 \pi]$, in a neighborhood of the point $x(d)$ slit along $\Gamma$. Consider polar coordinates $(|x-x(d)| \cos \varphi,|x-x(d)| \sin \varphi)$ in the neighborhood of $x(d)$. Assume that $\varphi \in(\alpha(d), \alpha(d)+2 \pi)$ if $d=a_{n}$ and $\varphi \in$ $(\alpha(d)-\pi, \alpha(d)+\pi)$ if $d=b_{n}$. Recall that $\alpha(s)$ is the angle between the direction of the axis $O x_{1}$ and the tangent vector $\tau_{x}$ to the contour $\Gamma$ at the point $x(s)$. Accordingly, 
$\alpha(d)=\alpha\left(a_{n}+0\right)$ if $d=a_{n}$, and $\alpha(d)=\alpha\left(b_{n}-0\right)$ if $d=b_{n}$. Thus, the angle $\varphi$ varies continuously in the neighborhood of the point $x(d)$ slit along the contour $\Gamma$. Denoting

$$
\mu_{1}^{d}(s)=\mu_{1}(s)|s-d|^{1 / 2}=\mathcal{Q}_{1 / 2}^{-1}(s) \mu_{1 *}(s)|s-d|^{1 / 2},
$$

we obtain at the end points of $\Gamma$ that $\mu_{1}^{d}(d)=\mu_{1}^{a_{n}}\left(a_{n}+0\right)$ if $d=a_{n}$ and $\mu_{1}^{d}(d)=$ $\mu_{1}^{b_{n}}\left(b_{n}-0\right)$ if $d=b_{n}$. Studying the behavior of $\nabla u\left[\mu_{1}, \mu_{2}\right](x)$ at the ends of the contour $\Gamma$ with the help of the technique developed in 1, 2, we arrive at the following statement.

Theorem 4. Let $x \rightarrow x(d) \in X$ and $x \notin \Gamma$. Then the following formula for the directional derivative of a solution to Problem $\mathbf{U}$ in the direction of $\mathbf{w}=(\cos \vartheta, \sin \vartheta)$ holds in the neighborhood of the point $x(d)$ :

$$
\begin{gathered}
\frac{\partial u\left[\mu_{1}, \mu_{2}\right]}{\partial \mathbf{w}}(x)=\frac{\mu_{1}^{d}(d)}{2|x-x(d)|^{1 / 2}} \cos \left(\vartheta-\frac{\varphi+\alpha(d)}{2}+\frac{\pi m}{2}\right) \\
-(-1)^{m} \frac{\mu_{2}(d)}{2 \pi}[\cos (\vartheta-\alpha(d)) \ln |x-x(d)|-\varphi \sin (\vartheta-\alpha(d))]+O(1),
\end{gathered}
$$

where $m=0$ if $d=a_{n}$ and $m=1$ if $d=b_{n} ; n=1, \ldots, N$. Here $O(1)$ denotes a function continuous at the point $x(d)$, as well as in its neighborhood slit along $\Gamma$.

It follows from Theorem 4, that, generally, derivatives of a solution to Problem $\mathbf{U}$ behave as $O\left(|x-x(d)|^{-1 / 2}\right)+O(\ln |x-x(d)|)$ near the end $x(d)$ of the contour $\Gamma$. However, if $\mu_{1}^{d}(d)=\mu_{2}(d)=0$, then the gradient of a solution $\nabla u\left[\mu_{1}, \mu_{2}\right](x)$ is bounded and even continuous both in the point $x(d)$ and in its neighborhood slit along $\Gamma$.

6. Asymptotic behavior of potentials at infinity. In this section we discuss asymptotic behavior of potentials $v\left[\mu_{1}\right](x)$ and $w\left[\mu_{2}\right](x)$ as $|x| \rightarrow \infty$. Let $\tau_{y}=$ $(\cos \alpha(\sigma), \sin \alpha(\sigma))$ and $\mathbf{n}_{y}=(\sin \alpha(\sigma),-\cos \alpha(\sigma))$ be tangent and normal vectors to $\Gamma$ at the point $y(\sigma) \in \Gamma$. Consider in the plane $R^{2}$ polar coordinates with the origin in the point $(0,0)$. Let the polar coordinates of a point $y(\sigma) \in \Gamma$ be $|y(\sigma)|$ and $\theta(\sigma)$. Let the polar coordinates of a point $x \in R^{2} \backslash \Gamma$ be $r=|x|$ and $\phi$. It follows from [6, section $1.5]$ that the asymptotic formulas

$$
\begin{gathered}
w\left[\mu_{2}\right](x)=\frac{i e^{-i \pi / 4}}{2 \sqrt{2 \pi k}} F_{1}(\phi) \frac{e^{i k r}}{\sqrt{r}}\left[1+O\left(\frac{1}{r}\right)\right], \quad r \rightarrow \infty, \\
v\left[\mu_{1}\right](x)=\frac{e^{-i \pi / 4}}{2} \sqrt{\frac{k}{2 \pi}} F_{2}(\phi) \frac{e^{i k r}}{\sqrt{r}}\left[1+O\left(\frac{1}{r}\right)\right], \quad r \rightarrow \infty,
\end{gathered}
$$

hold, where

$$
\begin{gathered}
F_{1}(\phi)=\int_{\Gamma} \mu_{2}(\sigma) e^{-i k|y(\sigma)| \cos (\theta(\sigma)-\phi)} d \sigma \\
F_{2}(\phi)=\int_{\Gamma} \rho\left[\mu_{1}\right](\sigma) \sin (\alpha(\sigma)-\phi) e^{-i k|y(\sigma)| \cos (\theta(\sigma)-\phi)} d \sigma .
\end{gathered}
$$

The asymptotic formulas hold both for the case $k=\operatorname{Re} k$ and for the case $\operatorname{Im} k>0$.

This research was partially supported by RFBR grants 08-01-00082, 09-01-00025. 


\section{REFERENCES}

[1] Krutitskii P.A. The Dirichlet problem for the Helmholtz equation outside slits in a plane. Comp. Maths. Math. Phys., 1994, v.34, No. 8/9, pp. 1073-1090. MR1300397 (95f:35046)

[2] Krutitskii P.A. The Neumann problem for the Helmholtz equation outside slits in a plane. Comp. Maths. Math. Phys., 1994, v.34, No. 11, pp. 1421-1431. MR.1307611(95i:45004)

[3] Krutitskii P.A. The mixed problem for the Helmholtz equation outside slits in a plane. Differential Equations, 1996, v.32, No.9, pp. 1153-1162. MR.1600844 (98m:35030)

[4] Krutitskii P.A. The modified jump problem for the Helmholtz equation. Ann. Univ. Ferrara, 2001, v. XLVII, pp. 285-296. MR1897572 (2003a:35037)

[5] Krutitskii P.A., Kolybasova V.V. A generalization of the Neumann problem for the Helmholtz equation outside slits on the plane. Differential Equations, Vol. 41, No. 9, 2005, pp. 1213-1224. MR2247253(2007c:35024)

[6] Galishnikova T.N., Il'inskii A.S. Numerical methods in problems of diffraction. Moscow: Publishing house of Moscow State University, 1987 (in Russian). MR898654 (88h:65230)

[7] Ih K.-D, Lee D.-J. Development of the direct boundary element method for thin bodies with general boundary condition. Journal of Sound and Vibration, 1997, v.202, pp. 361-373. MR.1454739 (98e:73144)

[8] Lifanov I. K. Method of singular integral equations and discrete vortices. VSP, Zeist, 1996. MR:1451377(98g:65130)

[9] Muskhelishvili N.I. Singular integral equations. Nauka, Moscow, 1968 (in Russian). English translation: Noordhoff, Groningen, 1972. MR0355494 (50:7968)

[10] Vladimirov V.S. Equations of mathematical physics. Nauka, Moscow, 1981 (in Russian). English translation: Mir Publishers, Moscow, 1984. MR764399 (86f:00030)

[11] Smirnov V.I. A course of higher mathematics. V. IV, V. Gostehizdat, Moscow-Leningrad, 1951 (in Russian). English translation: Pergamon Press, Oxford, 1964. MR0049255 (14:145e)

[12] Nikiforov A.F., Uvarov V.B. Special Functions of Mathematical Physics: A Unified Introduction With Applications. Nauka, Moscow, 1984 (in Russian). English translation: Birkhäuser, Basel, 1988. MR799713 (86k:33001)

[13] Krutitskii P.A. An explicit solution of the pseudo-hyperbolic initial boundary value problem in a multiply connected region. Math. Meth. Appl. Sci., 1995, v.18, pp. 897-925. MR.1346665 (97d:76010a)

[14] Kolmogorov A.N., Fomin S.V. Elements of function theory and functional analysis. Nauka, Moscow, 1981 (in Russian). English translation: Dover, New York, 1999. MR630899 (83a:46001)

[15] Mikhlin S.G. Course of mathematical physics. Nauka, Moscow, 1968 (in Russian). English translation: North-Holland Publishing Co., Amsterdam-London, 1970.

[16] Vinogradov I.M. (editor). Encyclopaedia of mathematics. V.1. Sovetskaya entsiklopediya, Moscow, 1977 (in Russian). English translation: Kluwer, Dordrecht, 1988. MR470361 (81c:00008a)

[17] Krein S.G. (editor). Functional analysis. Nauka, Moscow, 1964 (in Russian). English translation: Wolters-Noordhoff Publishing, Groningen, 1972.

[18] Kantorovich L.V., Akilov G.P. Functional Analysis. Nauka, Moscow, 1984 (in Russian). English translation: Pergamon Press, Oxford, 1982. MR664597 (83h:46002)

[19] Trenogin V.A. Functional Analysis. Nauka, Moscow, 1980 (in Russian). French translation: Mir Publishers, Moscow, 1985. MR836334 (87g:46001)

[20] Krutitskii P.A. Boundary value problem for the Helmholtz equation outside slits in a plane with the boundary conditions of the third kind. Differential equations, 2007, v. 43, No. 10, pp.1387-1399.

[21] Wolfe P. An existence theorem for the reduced wave equation. Proc. Amer. Math. Soc., 1969, v.21, pp.663-666. MR0265780 (42:689)

[22] Lifanov I.K., Poltavskii L.N., Vainikko G.M. Hypersingular integral equations and their applications. CRC Press, Boca Raton, 2004. MR2053793 (2005e:45001) 